\title{
ON THE COMPLETE INTEGRAL CLOSURE OF A DOMAIN
}

\author{
PAUL HILL ${ }^{1}$
}

\begin{abstract}
For a given positive integer $n$, a semivaluation domain $D_{n}$ is constructed so that the complete integral closure has to be applied successively exactly $n$ times before obtaining a completely integrally closed domain. Letting $G_{n}$ be the group of divisibility of $D_{n}$, we set $G=\Sigma ⿴ 囗 十 G_{n}$, the cardinal sum of the groups $G_{n}$. It is concluded that the semivaluation domain $D$ having $G$ as its group of divisibility is a Bezout domain with the property that $D \subset D^{*} \subset D^{* *} \subset D^{* * *} \subset \cdots$ is a strictly ascending infinite chain, where $D^{*}$ is the complete integral closure of $D$.
\end{abstract}

By a classical theorem of Krull, if $G$ is a totally ordered abelian group then there exists a valuation domain $D$ having $G$ as its group of divisibility. There is, in fact, a standard construction of such a domain $D$ based on the group algebra $k(G)$ over any field $k$. This construction was generalized from the case that $G$ is totally ordered to a lattice ordered group $G$ by P. Jaffard in [2]. Thus if $G$ is an abelian l-group, we have via Krull-Jaffard an integral domain $D[G]$ whose group of divisibility is $G$. J. Ohm observed in [3] that this semivaluation domain $D[G]$ is always a Bezout domain. The terminology "semivaluation domain" was established in [4].

The complete integral closure of a domain $D$ in its quotient field is denoted herein by $C(D)$ (a domain $R$ is completely integrally closed if $R$ contains each element $x$ of its quotient field for which there exists an element $d \neq 0$ in $R$ such that $d x^{n} \in R$ for all $n \geqq 0$ ). More generally, for any positive integer $n$, let $C^{n}(D)=C\left(C^{n-1}(D)\right)$. In [1], W. Heinzer gave an example of a lattice ordered abelian group $G$ such that $C^{2}(D[G]) \neq$ $C(D[G])$. Thus, $D[G]$ in Heinzer's example is a Bezout domain whose complete integral closure is not completely integrally closed. In Heinzer's example, $D[G]$ has infinite dimension, but $\mathrm{P}$. Sheldon $[5]$ has refined the example so that $D[G]$ has dimension two.

Received by the editors December 2, 1971 .

AMS 1970 subject classifications. Primary 13B20; Secondary 06A60.

Key words and phrases. Complete integral closure, semivaluation, Bezout domain, group of divisibility, lattice ordered group.

${ }^{1}$ This research was supported in part by NSF Grant GP-29025.

(c) American Mathematical Society 1972 
Although we have examples where $C^{2}(D[G]) \neq C(D[G])$, it has not been known whether or not the inequality can persist between $C^{n}(D[G])$ and $C^{n+1}(D[G])$ for $n>1$. In particular, we have the question concerning the complete integral closure of $C^{2}(D[G])$. In the examples of Heinzer and Sheldon, $C^{2}(D[G])$ is completely integrally closed, that is, $C^{2}(D[G])=$ $C^{3}(D[G])$. However, our purpose is to show that there exists a Bezout domain $D$ such that the operation of taking successively the complete integral closure beginning with $D$ does not stabilize in a finite number of steps. Since the domain $D$ with the desired properties turns out to be $D[G]$ for some l-group $G$, our interest is focused on $G$.

The desired lattice ordered group will be built in stages. The first component is closely related to Heinzer's example in [1]. Let $I \oplus J$ denote the lexicographic sum of two copies of the integers; $(i, j) \geqq 0$ if $i \geqq 1$ or if $i=0$ and $j \geqq 0$. Let $G_{1}$ denote the collection of all functions $f$ from the set $N$ of nonzero integers into $I \oplus J$ that satisfy the following conditions. If $f(n)=$ $(i(n), j(n))$, then

(1) $i(n)=0$ whenever $n$ is negative.

(2) $i(n)=0$ for all but a finite number of positive $n$.

(3) $j(n)=0$ for all but a finite number of negative $n$, but there is no condition on $j(n)$ for positive $n$.

The set $G_{1}$ is a group with respect to pointwise addition. Furthermore, if we make $f \geqq 0$ in $G_{1}$ if and only if $f(n) \geqq 0$ for each $n \in N$, then $G_{1}$ is endowed with a lattice order. Before we describe $G_{2}$, two convex $l$ subgroups of $G_{1}$ are introduced. Let

$$
A=\left\{f \in G_{1}: f(n)=(i(n), j(n)), \text { where } j(n)=0\right.
$$

for all but a finite number of $n \in N\}$,

and let

$$
\begin{aligned}
K=\left\{f \in G_{1}: f(n)=\right. & (i(n), j(n)), \text { where } i(n)=0 \\
& \text { for all } n, j(n)=0 \text { if } n<0, \text { and } j(n)=0 \\
& \text { for all but a finite number of positive } n\} .
\end{aligned}
$$

We now proceed to the construction of $G_{2}$. Let $P=P_{1}+P_{2}+\cdots+P_{k}+\cdots$ be a partition of the positive integers into an infinite number of infinite disjoint subsets $P_{k}$. Define the $l$-subgroup $G_{2}$ of $G_{1}$ by

$$
\begin{gathered}
G_{2}=\left\{f \in G_{1}: \text { if } f(n)=(i(n), j(n)) \text {, then }(1) j(n)\right. \\
\text { is constant on } P_{k} \text { for all but a finite number of } \\
k \text {, and (2) there exists, for each } k \text {, integers } \\
r \text { and } s \text { such that } j(n)=r n+s \\
\text { for all but a finite number of } \left.n \text { in } P_{k}\right\} .
\end{gathered}
$$

One of the most interesting features of $G_{2}$ is that $G_{2} / K$ is lattice isomorphic to $G_{1}$ under a mapping $\phi: G_{1} \rightarrow G_{2} / K$ defined as follows. If $f \in G_{1}$ and if 
$f(n)=(i(n), j(n))$, then $\phi(f)=g+K$, where $g(n)=(\alpha(n), \beta(n))$ with $\alpha(n)$ and $\beta(n)$ being defined by:

$$
\begin{array}{ll}
\alpha(n)=0 & \text { if } n \text { is negative, } \\
\alpha(n)=j(-2 n) & \text { if } n \text { is positive, } \\
\beta(n)=j(2 n+1) & \text { if } n \text { is negative, } \\
\beta(n)=n i(k)+j(k) & \text { if } n \in P_{k} .
\end{array}
$$

It is routine to verify that $\phi$ is an isomorphism from $G_{1}$ onto $G_{2} / K$. In order to show that $\phi$ is an $l$-isomorphism, it suffices to show that $\phi(f) \geqq 0$ if and only if $f \geqq 0$. In this connection, recall that $\phi(f)=g+K \geqq 0$ in $G_{2} / K$ if and only if $g+k \geqq 0$ in $G_{2}$ for some $k \in K$. Now suppose that $f \geqq 0$ in $G_{1}$. Then $f(n)=(i(n), j(n)) \geqq 0$ in $I \oplus J$ for each nonzero integer $n$. It follows that $i(n) \geqq 0$ for all $n \in N$ and that $j(n) \geqq 0$ for all negative $n$ since $i(n)=0$ if $n<0$. Furthermore, if $j(n)<0$ for a positive $n$, then $i(n)>0$. Thus if $\beta(n)=$ $n i(k)+j(k)<0$ for $n \in P_{k}$, then $i(k) \neq 0$. Therefore, $i(k)>0$ and $\beta(n) \geqq 0$ for all but a finite number of $n \in P_{k}$. Since $i(k)=0$ for all but a finite number of $k$, we conclude that $\beta(n)<0$ for at most a finite number of positive $n$. Since $K$ absorbs the components $\beta(n)$ for a finite number of positive $n$, it follows that $\phi(f)=g+K=(\alpha(n), \beta(n))+K \geqq 0$. The argument that $\phi(f) \geqq 0$ implies that $f \geqq 0$ follows from a similar but somewhat simpler analysis.

It is important to observe that $\phi(A) \supseteq A \mid K$, where $A$ and $K$ are the subgroups of $G_{1}$ introduced earlier. In particular, $A \subseteq G_{2}$. Letting $\beta: G_{2} \rightarrow$ $G_{2} / K \gg^{\phi^{-1}} G_{1}$ be the composition of the natural map $G_{2} \rightarrow G_{2} / K$ and the $l$-isomorphism $\phi^{-1}$, we have the exact sequence

$$
K \stackrel{i}{\longrightarrow} G_{2} \stackrel{\beta}{\rightarrow} G_{1},
$$

where $i$ is the inclusion map and $\beta$ is an $l$-homomorphism. We define $G_{n}$ for $n \geqq 3$ inductively by $G_{n+1}=\beta^{-1}\left(G_{n}\right)$, the complete inverse image of $G_{n}$ under $\beta$. Since $\beta$ is an $l$-homomorphism, $G_{n+1}$ is an $l$-subgroup of $G_{n}$ and $G_{n+1} / K$ is $l$-isomorphic to $G_{n}$. Furthermore, since $\phi(A) \supseteq A / K$, we see that $\beta(A) \subseteq A$. Hence $A \subseteq G_{n}$, for each $n$, inductively.

Recall that an element $b \geqq 0$ in any lattice ordered abelian group $G$ is said to be a bounded element of $G$ if there exists $g \in G$ such that $n b \leqq g$ for each positive integer $n$. The bounded elements of $G$ form a convex subsemigroup of $G$, and the group

$$
B(G)=\{x: x=b-c \text { where } b \text { and } c \text { are bounded in } G\}
$$

is an ideal of $G$. As is well known, there is a connection between bounded elements and the complete integral closure. If the Bezout domain $D$ has $G$ for its group of divisibility, then its complete integral closure $C(D)$ has 
$G / B(G)$ for its group of divisibility. In particular, if $G$ is any abelian $l$ group and $D=D[G]$ is the semivaluation domain associated with $G$, then $C(D)$ has $G / B(G)$ for its group of divisibility. In view of this, we are especially interested in $B\left(G_{n}\right)$ for the $l$-groups $G_{n}$ that we have constructed. A simple inspection reveals that $B\left(G_{1}\right)=K=B(A)$, and therefore $B\left(G_{n}\right)=K$ for all $n$ since $G_{1} \supseteq G_{n} \supseteq A$.

We have essentially established our first main result.

THEOREM 1. For any nonnegative integer $n$, there exists a semivaluation domain $D\left[G_{n}\right]$ such that

$$
C\left(D\left[G_{n}\right]\right) \subset C^{2}\left(D\left[G_{n}\right]\right) \subset \cdots \subset C^{n+1}\left(D\left[G_{n}\right]\right)=C^{n+2}\left(D\left[G_{n}\right]\right)=\cdots .
$$

Proof. Suitable examples $G_{0}$, for $n=0$, are in abundance. For example, we can take $G_{0}=A \subseteq G_{1}$. If $n>0$, we shall show that the $l$-group $G_{n}$ constructed above satisfies the condition of the theorem. We have observed that $G\left(B_{1}\right)=K$, but it is easy to verify that $B\left(G_{1} / K\right) \neq 0$, whereas $B\left(\left(G_{1} / K\right) / B\left(G_{1} / K\right)\right)=0$. We conclude that $C\left(D\left[G_{1}\right]\right) \subset C^{2}\left(D\left[G_{1}\right]\right)=$ $C^{3}\left(D\left[G_{1}\right]\right)$. The theorem now follows inductively from the $l$-isomorphism $G_{n+1} / K \cong G_{n}$ and the equation $B\left(G_{n+1}\right)=K$.

We see from Theorem 1 that, for any prescribed positive integer $n$, there exists a semivaluation domain $D$ such that the complete integral closure has to be applied exactly $n$ times before obtaining a completely integrally closed domain. The next theorem, however, shows that there exists a semivaluation domain $D$ such that $C^{n}(D)$ is not completely integrally closed for any $n$.

THEOREM 2. There exists a (Bezout) semivaluation domain $D$ such that the infinite sequence

$$
D \subset C(D) \subset C^{2}(D) \subset \cdots \subset C^{n}(D) \subset \cdots
$$

is strictly ascending.

Proof. Choose the $l$-groups $G_{n}$ so that they satisfy the conditions of Theorem 1. Let $G=\sum_{n \geqq 0} \boxplus G_{n}$ be the (small) cardinal sum of the groups $G_{n}$. Letting $\bar{B}(G)=G / B(G)$ and letting $\bar{B}^{k+1}=\bar{B} \bar{B}^{k}$, we see that

$$
\bar{B}^{k}(G)=\sum_{n \geqq 0} \boxplus \bar{B}^{k}\left(G_{n}\right)
$$

since $\bar{B}$ commutes with cardinal sums. Since $\bar{B}^{k}\left(G_{n}\right)$ is different from zero if $k<n+1, \bar{B}^{k}(G)$ is different from zero for all $n$. Hence $C^{k}(D[G])$ is not completely integrally closed no matter what $k$ is. 


\section{REFERENCES}

1. W. Heinzer, Some remarks on complete integral closure, J. Austral. Math. Soc. 9 (1969), 310-314. MR 40 \#4254.

2. P. Jaffard, Contribution à l'étude des groupes ordonnés, J. Math. Pures Appl. (9) 32 (1953), 203-280. MR 15, 284.

3. J. Ohm, Some counterexamples related to integral closure in $D[[X]]$, Trans. Amer. Math. Soc. 122 (1966), 321-333. MR 34 \#2613.

4. - Semi-valuations and groups of divisibility, Canad. J. Math. 21 (1969), 576591. MR 39 \#4146.

5. P. Sheldon, A counterexample to a conjecture of Heinzer (preprint).

Department of Mathematics, Florida State University, Tallahassee, Florida 32306 